\title{
Note ON APPEARANCE Of ZigZaK TyPe Self Similarity IN Flying Bird Flocks Performing Directional Collective Motions in Mild-Weather CONDITIONS
}

\author{
ADAM GADOMSKI ${ }^{1}$, JANUSZ CYRAN ${ }^{2}$ \\ ${ }^{1}$ Department of Physics, Institute of Mathematics and Physics, UTP University of Science \& Technology, al. Kaliskiego 7, \\ PL-85796 Bydgoszcz, Poland; agad@utp.edu.pl \\ ${ }^{2}$ Programming Laboratory Lobster, Zebrzydowicka 10, PL-44210 Rybnik, Poland; januszcyran@gmail.com
}

Received January 05, 2018; accepted May 07, 2018; published online May 25, 2018

\begin{abstract}
It is argued here that very regular and coordinated patterns of bird flocks in motion, in quiescent weather conditions, can be expected to first emerge upon minimal configurational entropy production for the flock(s) vs. air mass couple. This is immediately to accept if certain ultralow friction conditions apply, with the air-bird flock friction coefficient $f$ approaching minimal values. Then, quite geometrically coordinated, apparently self-similar zigzak type patterns show up during the flock's motion. Otherwise, the coherence of the flying patterns is lost, the flocks become disorganized, characteristic of fairly large configurational-space (individual positions involving) entropy, and their average acceleration supposedly gets on appreciable nonzero values. The oversimplified analysis performed in this study does not assume really far-from-equilibrium (thermo)dynamic conditions to occur.
\end{abstract}

\section{INTRODUCTION}

Birds and their flocks have been the subject of many speculations and controversial debates over many years. A bird, as an individual when flying, is commonly described, as a creature appealing to human imagination more in terms of a natural airplane albeit such a means of thinking ought rather to be reversed since the airplane is after all a standard example of outstanding and sophisticated bionic solution (Vincent et al. 2006). Yet, the flight of an individual bird is recently going to be not of really utmost concern, because rather collective motions of birds are more challenging due to many reasons (Vicsek \& Zafeiris, 2012). (Hereafter, the birds while in motion/flight are dealt with as a physical, in particular, a thermodynamic system.)

First, our curiosity can be expressed in terms of whether such motions are more performed by chance or the chance has to be of negligible magnitude for their final outcomes?

Second, to what extent keeping the direction by a flock of birds is under the bird-group control, if it could really be the case, or the whole directional performance of the formation is induced by external factors? These factors can be, for example, distinctive changes in weather's attitude or (almost) random drastic events, emerging suddenly in the surroundings, such as extremal actions of humans, e.g. due to explosions, massive airplanes' spectacles, over-pollutions, military and/or hunting actions, and the likes (Sornette, 1999).

One may also accept that, except of keeping certain directional motions by the flocks, an adequate separation distance between the individuals, constituting the overall bird flock under discussion, has to be indicated as the basic property of the flock while in motion, Figures 1-3. Next, a tendency of the flying pattern to be in cohesion conditions, thus keeping altogether the individuals of the formation, alias, expecting the external behavior of the pattern in motion to go repeatedly, and roughly, in a self-similar (local-versus-global) predictive fashion, see Figures 1-3, has to be, in our opinion, a marked signature of the flock-in-flight behavior (Gadomski \& Siódmiak, 2013).

Summing up, in part, random vs. deterministic expression of the bird flocks' long-migration-distance involving motions, and in addition, three basics named: assumed motion directionality; adequate-distance separation between the individuals while in motion plus a certain necessary cohesiveness as to keep the flock as if it was one body (Vicsek \& Zafeiris, 2012; Gadomski \& Siódmiak, 2013), would be principal behavioral landmarks for accomplishing proper description of their complex total motion. Nature is the best ever laboratory to perform albeit preliminary yet intuitively decisive 
observations on collective groups' motions, a perennially alive subject of studies.

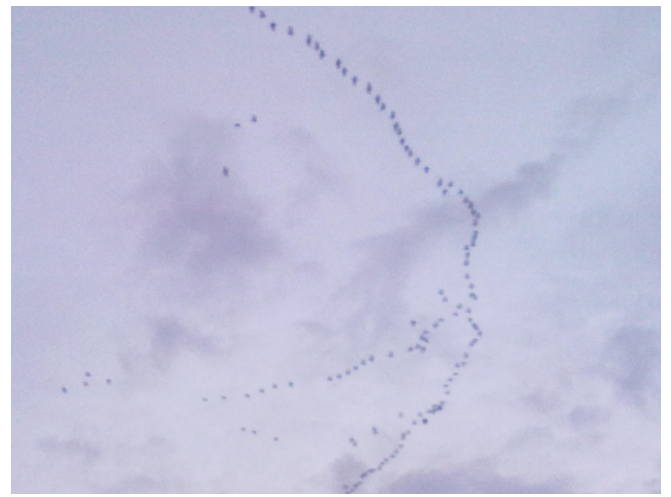

Fig. 1. A first group of birds (while in its directional motion) described in the Text, showing up signatures of right-angle and overall arrow-like (straight-line addressing) emergent, mesoscopic (Laughlin et al., 2000) 'nonlinear' patterns, accommodating to certain mild-weather local wind-, temperature- and respective humidity involving conditions in a region located in northern part of Poland

\section{NAÏVE-OBSERVER DISCUSSION}

The goal of this short descriptive note (or, a brief essay) is to draw one's attention and intuition to attempt on observing nature's phenomena appearing around us. In the following, in seemingly qualitative terms, we wish to present three interesting pictures of real bird flocks, taken a few years ago in a northern part of Poland during evening-weather very mild and weak-wind conditions at the ambient temperature at about eight Celsius grades.

The first bird-motional pattern taken, Figure 1, containing about one hundred of birds, reveals a temporary performance by the flight toward some geographical direction that might be for the moment of secondary importance. At the instance of taking the picture, however, the direction is to be indicated as the eastern one. As one can see from the pattern in Fig. 1, the birds are first aligned in the flock's front. Then, two tails of the remaining bird "constituents", join the front apparently in a perpendicular way. The flock while in motion splits somehow into two, three or several additional sub-flocks (dynamic clusters), involving several other birds (per sub-flock), participating in the overall directional motion.

Then, after the first flock described, a second one came after less than one minute for taking it in a form of Figures 2 and 3, respectively. During the evening conditions taking pictures is somehow a hazardous job but it has been succeeded (in a simple technical way) to take the visible enough patterns, thus, eventually worth analyzing.

Namely, on Fig. 2 one can observe that the bird flocks develop the aligned patterns that at their temporarily formed wedges constitute a local zigzak emergent pattern quite characteristic of a right-angle (apparently, of ca. 90 grades) value. Moreover, the overall pattern is also reproduced by some perhaps slightly delayed individuals, following the main front, with a similar right-angle signature kept while performing the subsequent motion, very likely due to the fact that the wedge just formed cuts optimally the really mild though opposing air mass, causing the efficient, i.e. constant, flock's mass-center velocity, $V$ (Vicsek \& Zafeiris, 2012; Gadomski \& Siódmiak, 2013) to be put otherwise under considerable challenge, when the conditions were suddenly changed.

Assumed that the flock is big enough, in terms of its individual constituents, and can be treated in an oversimplified way as one body decelerated by the air mass, thus, being opposed with the friction coefficient $f$ by the attacking air flow, one can easily discover a formula for the average flock's acceleration $a$ upon changing flight's conditions due to an external cause. It reads:

$$
a=f g h,
$$

wherein $g$ is the gravity constant roughly of $10[\mathrm{~N} / \mathrm{kg}]$, and $h$ is a (dimensionless) ratio of characteristic linear dimensions of the flock such as a mean between-twobirds separation distance (local measure; denoted by $l$ ) as related to its configurational spanning distance, a global measure to be taken from vectorial analysis of the flock's configuration at the time of evolution change; designated by $L$. (Thus, one gets $h=l / L$, which can be indicated as a characteristic bird-flock geometrical parameter.) The distance $L$ can be proposed as a measure of the configurational flocks entropy (or, 
coordination efficiency). Such a simple measure of $a$ from Eq. (1) can be justified upon assuming that the flock, when flying, always tends to achieve minimum of its inertia (temporal) change, i.e. $d I / d t$ (Gadomski \& Siódmiak, 2013) with $t$ - time, a quantity proportional to the entropy production change (Laughlin et al., 2000), $d S / d t$ for the flock-air dynamic couple, also provided as virtually minimal, if the flight is expected to go smoothly, namely:

$$
d I / d t \sim d S / d t
$$

Both rates involved in Eq. (2) are proportional to $V$ (mass-center velocity) but $d I / d t=M(l+L) V$ applies, where $M$ stands for the total flock's mass. When the individual temporal velocities of the birds in flock adjust fairly well to $V$ one may foresee that all the kinetic energy terms embark on almost very same values, and the only dissipation-energy term that remains operative is the "thermodynamic" acceleration $a$ from Eq. (1), to be manifested upon each change of the flock's direction. Then, upon quiescent flock-air (thermo)dynamic conditions, let us say the ones of Fig. 3, the flock's movement proceeds with constant velocity $(V)$, and minimum kinetic-thermodynamic (the opposing air intrudes minimally to the system) energy loss if the acceleration a quickly approaches zero which is true upon reaching minimal values by the friction term $f$ from Eq. (1), cf. (Scherge \& Gorb, 2001). In such conditions of $f \sim 0$ self-similar patterns, with nearly right-angle geometry embodied in their very coordinated configuration, can be reproduced, cf. Fig. 2 and Fig. 3. Note that Eq. (1) is possible to derive upon least dissipation conditions (Scherge \& Gorb, 2001). This is equivalent to accept that the work needed to optimally span the birds configuration upon flying would approximate the least flock's disorganization heat effect with $f \sim 0$, but with a balanced account of all kinetic energy terms involved in flock's motion. The thermodynamic driving force's account pertinent to this approach is closely related to the overall energy account allowing the system to keep an adequate separation distance between any pair of birds in the flying flock, thus involving $l$ as related to $L$ in favor of $l<<L$, in the constant velocity $(V)$ limit.

It is really seen, and very expressed by this natural example, that to perform such type of collective and also coordinated motion, the pattern has apparently to split into simple self-similar patterns (or, quasi fractal-like) both within a suitable match of the global as well as local space scales, cf. Fig. 2.

It is seen even more adequately, when looking carefully at Fig. 3 in which the basic right-angle zigzak type pattern is naturally reproduced during such quiescent local-weather and peculiar conditions (with $f$, being also a dimensionless quantity, here approaching zero-value limit) close to Bydgoszcz, Kujawy and Pomorze Region in Poland, by that date of examination (November 13, 2013), also verifiable at the corresponding link http://www.weather-forecast.com/ locations/Bydgoszcz/forecasts/latest.

Table 1. Juxtaposition of main qualitative observations

\begin{tabular}{cccc}
\hline Observations & $\begin{array}{c}\text { (Non)linear } \\
\text { qualitative feature }\end{array}$ & $\begin{array}{c}\text { Branching and } \\
\text { angular property }\end{array}$ & $\begin{array}{c}\text { Number of patterns and } \\
\text { coherence conditions }\end{array}$ \\
\hline $\begin{array}{l}\text { Fig. 1: main points } \\
\text { Fig. 2: main points }\end{array}$ & $\begin{array}{c}\text { Pronounced } \\
\text { Mild }\end{array}$ & $\begin{array}{l}\text { Present } \\
\text { branches/right-angle } \\
\text { trend } \\
\text { Fairly right-angle }\end{array}$ & $\begin{array}{c}\text { Few; noncoherent } \\
\text { Fairly coordinated } \\
\text { behavior }\end{array}$ \\
Fig. 3: main points & Weakened & $\begin{array}{c}\text { Few; fairly coherent } \\
\text { right-angle multiplication } \\
\text { (self-similar) }\end{array}$ \\
\hline
\end{tabular}

\section{RESUME}

To recap, cf. Table 1, it has been argued in fairly qualitative terms only, and in accord with the basic knowledge accumulated in (Vicsek \& Zafeiris, 2012), that under certain realistic local-weather conditions, a certain precisely determinable self-similar, zigzak, rightangle and emergent nonequilibrium pattern, upon following a bird flock while in directional motion, is feasible to disclose. As described, the appearance ought to be adequately explained in terms of bird-flock selforganization as a dynamic event, conforming to its respective local-weather conditions, these peculiar dynamics also apparently observed in diffusion-reaction systems, driven out of thermodynamic equilibrium, see (Lemarchand \& Nowakowski, 1998) for example. The dynamics of self-organization observed when following flocks on the sky is corroborated by the organizational self-similar patterns of the virtually greatest efficiency of right-angle geometrical character here. Presenting just on purpose the core components of self-organization as a far-from-equilibrium process (Lemarchand \& Nowakowski, 1998), and merging it for a while with soexpressed geometry, one may speak of a certain non- 
grand unification of those two fields of physics, not mentioning that (birds') kinematics is always depicted in terms of geometry complemented by the time variable: the motions go over an observation time span for sure. Or, when anticipating many other circumstances, e.g. those concerning ecological systems, especially the ones with velocity alignment, cf. (Romanczuk \& Schimansky-Geier, 2012), presumably with some distinctive involvement of stochastic force fields of space-and-time (un)correlated propensity for which Eq. (1) with $f \sim 0$ stands only for a crude linear approximation (Vicsek \& Zafeiris, 2012; Laughlin et al., 2000). For natural systems approaching $f \sim 0$ one is encouraged to see (Scherge \& Gorb, 2001).

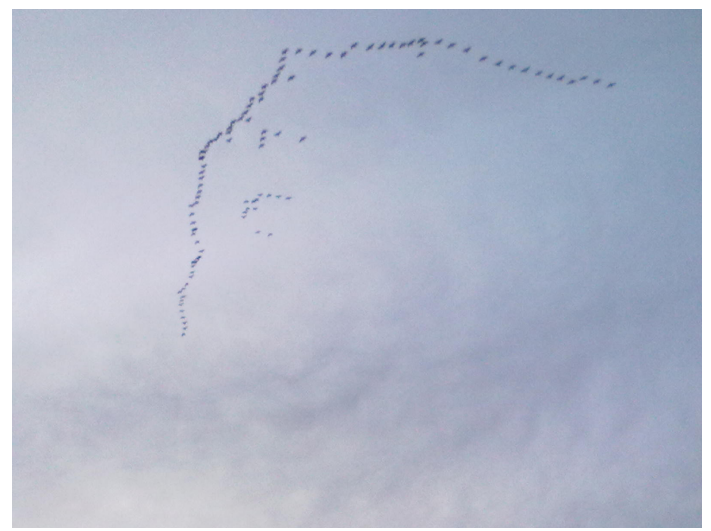

Fig.2. A (picturesque) evidence for the self-similar or "fractallike" overall nearly right-angle zigzak pattern, manifested at the fronts' wedges, and thus, characteristic of bird flocks' destination flights in mild-weather conditions

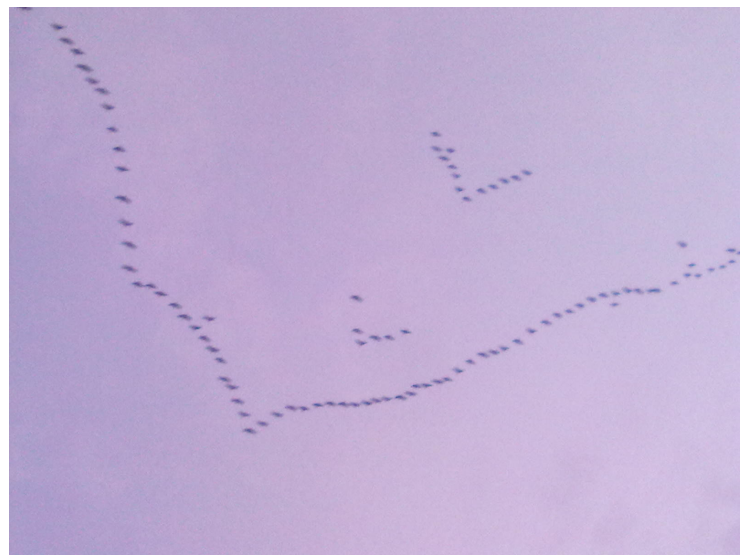

Fig.3 An eye-observer viz qualitative evidence for the selfsimilar overall (nearly) right-angle zigzak natural coordinated pattern of the same flying bird flock, compare with Fig. 2, with even more distinctly visible self-similar, repeatable and emergent objects of interest.

To assess in simple quantitative terms the principia of birds' flights, one has either to resort to tedious numerical analyses (Vicsek \& Zafeiris, 2012) or to accept certain oversimplified elucidations based on Newton's laws and the flock's inertia concept, addressing its minimization conditions over flight-time or evolution domain. The latter assumes, however, that the flock can be treated as one-body system, upon approaching a good enough number of its "constituents" (birds), thus, it goes smoothly for really large flocks (Gadomski \& Siódmiak, 2013), and can only be a crude, close-to-equilibrium approximation of the quite popular, emergent behavior (Laughlin et al., 2000) presented here.

To sum up, it is worth expressing in clear and detailed enough terms our argumentation about applying the ideas of non-equilibrium thermodynamics for the dynamic system of birds. Thus, let us explain why thermodynamics may work of the bird flocks by systematically juxtaposing the main arguments advocating for these points, and being underscored in the preceding section: (i) our main argument concerns the frictional viz dissipative interaction of the flock with the external air phase of varying thermodynamics (wind directions and mass-air intensity, air humidity, pressure vs. temperature conditions, lightening and ionization effects, etc.); (ii) in the most simplified and primitive case the flock can be treated as the quasi-rigid body that rubs case-sensitively over the masses of air; (iii) the least dissipation conditions are expressed by encountering the most optimal thermodynamicgeometric requirements, resulting in acceleration $a$ of Eq. (1) tending to zero, and implying that the coefficient of friction $f$ - itself a thermodynamic variable - is approaching a minimal value (ideally, very close to zero) as the geometric $h$-value would obey $h<<1$; (iv) the latter via the separation length, $l$, does tacitly contribute to the minimum entropy production by means of Eq. (2) which assumes similar behavior of the entropy production and mechanical condition of minimal inertia rate change upon rearranging the flock's finite and linearized structure $(l<<L$ or $h<<1)$ when meeting the resistive air-mass during the overall flock's motion. Such scenario drawn is reminiscent of directed percolation non-equilibrium phenomena after surpassing a (thermodynamic) critical point, cf. Hinrichsen, 2000. It also resembles certain but differently comprehended swarms addressing behaviors or locust involving dynamics in which, however, rather direction imposing mechanical nearest neighborhood (no-turn-back, cf. Romanczuk and Schimansky-Geier, 2012) correlated requirements are prevailing against after-criticality phase transitional out-of-equilibrium thermodynamic drives (Hinrichsen, 2000; Vicsek and Zafeiris, 2012).

\section{ACKNOWLEDGMENTS}


One of us (AG) is indebted to a very friend from his childhood (Krzysztof) for bearing to him in a very consequent way some very simple stork-flock type pictures from the past. The overall (thermo)dynamic picture achieved in this study has also been invented after watching a movie on birds in flight generously offered by Jacek in Bielsko-Biała. Technical support of Mrs. H. Przewoźniak (UTP) is appreciated through BS $39 / 14$.

\section{REFERENCES}

Gadomski A. \& Siódmiak J. (2013) Biophysics, Publishing Dept. of the UTP University of Science \& Technology, Bydgoszcz, chap. 1 (in Polish).

Hinrichsen H. (2000) Non-equilibrium critical phenomena and phase transitions into absorbing states, Adv. Phys. 49, 815958.

Laughlin R.B., Pines D., Schmalian J., Stojkovic B.P. \& Wolynes P. (2000) The middle way. Proc. Natl. Acad. Sci. USA. 97, 32-37.

Lemarchand A. \& Nowakowski B. (1998) Perturbation of local equilibrium by a chemical wave front, J. Chem. Phys. 109, 7028.

Romanczuk P. \& Schimansky-Geier L. (2012) Mean-field theory of collective motion due to velocity alignment, Ecological Complexity 10, 83-92.

Scherge M. \& Gorb S. (2001) Biological micro- and nanotribology. Nature's solutions. Springer, Berlin.

Sornette D. (1999) Complexity, catastrophe and physics, Physics World 12, 57.

Vicsek T. \& Zafeiris A. (2012) Collective motion, Phys. Rep. $\mathbf{5 1 7}, 71-140$.

Vincent J.F.V., Bogatyreva O.A., Bogatyrev N.R., Bowyer A., Pahl A.K. (2006) Biomimetics: its practice and theory, J. Royal Soc. Interface 3, 471-482. 